\title{
RESEARCH
}

Open Access

\section{Differential chemokine alteration in the variants of primary progressive aphasia-a role for neuroinflammation}

\author{
Aitana Sogorb-Esteve ${ }^{1,2}$, Imogen J. Swift ${ }^{1,2}$, Ione O. C. Woollacott ${ }^{2}$, Jason D. Warren ${ }^{1}$, Henrik Zetterberg ${ }^{1,3,4}$ and \\ Jonathan D. Rohrer ${ }^{2^{*}}$ (D)
}

\begin{abstract}
Background: The primary progressive aphasias (PPA) represent a group of usually sporadic neurodegenerative disorders with three main variants: the nonfluent or agrammatic variant (nfvPPA), the semantic variant (svPPA), and the logopenic variant (IVPPA). They are usually associated with a specific underlying pathology: nfvPPA with a primary tauopathy, svPPA with a TDP-43 proteinopathy, and IVPPA with underlying Alzheimer's disease (AD). Little is known about their cause or pathophysiology, but prior studies in both AD and svPPA have suggested a role for neuroinflammation. In this study, we set out to investigate the role of chemokines across the PPA spectrum, with a primary focus on central changes in cerebrospinal fluid (CSF)

Methods: Thirty-six participants with sporadic PPA (11 SvPPA, 13 nfvPPA, and 12 IVPPA) as well as 19 healthy controls were recruited to the study and donated CSF and plasma samples. All patients with IvPPA had a tau/Aß42 biomarker profile consistent with AD, whilst this was normal in the other PPA groups and controls. We assessed twenty chemokines in CSF and plasma using Proximity Extension Assay technology: CCL2 (MCP-1), CCL3 (MIP-1a), CCL4 (MIP-1ß), CCL7 (MCP-3), CCL8 (MCP-2), CCL11 (eotaxin), CCL13 (MCP-4), CCL19, CCL20, CCL23, CCL25, CCL28, CX3CL1 (fractalkine), CXCL1, CXCL5, CXCL6, CXCL8 (IL-8), CXCL9, CXCL10, and CXCL11.

Results: In CSF, CCL19 and CXCL6 were decreased in both svPPA and nfvPPA compared with controls whilst CXCL5 was decreased in the nfvPPA group with a borderline significant decrease in the svPPA group. In contrast, CCL2, CCL3 and CX3CL1 were increased in IVPPA compared with controls and nfvPPA (and greater than SVPPA for CX3CL1). CXCL1 was also increased in IVPPA compared with nfvPPA but not the other groups. CX3CL1 was significantly correlated with CSF total tau concentrations in the controls and each of the PPA groups. Fewer significant differences were seen between groups in plasma, although in general, results were in the opposite direction to CSF, i.e. decreased in IVPPA compared with controls (CCL3 and CCL19), and increased in svPPA (CCL8) and nfvPPA (CCL13).
\end{abstract}

\footnotetext{
* Correspondence: j.rohrer@ucl.ac.uk

${ }^{2}$ Dementia Research Centre, Department of Neurodegenerative Disease, UCL

Queen Square Institute of Neurology, University College London, London

WC1N 3BG, UK

Full list of author information is available at the end of the article
}

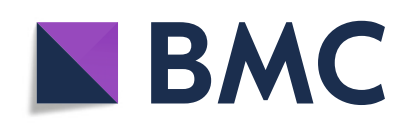

(c) The Author(s). 2021 Open Access This article is licensed under a Creative Commons Attribution 4.0 International License, which permits use, sharing, adaptation, distribution and reproduction in any medium or format, as long as you give appropriate credit to the original author(s) and the source, provide a link to the Creative Commons licence, and indicate if changes were made. The images or other third party material in this article are included in the article's Creative Commons licence, unless indicated otherwise in a credit line to the material. If material is not included in the article's Creative Commons licence and your intended use is not permitted by statutory regulation or exceeds the permitted use, you will need to obtain permission directly from the copyright holder. To view a copy of this licence, visit http://creativecommons.org/licenses/by/4.0/ The Creative Commons Public Domain Dedication waiver (http://creativecommons.org/publicdomain/zero/1.0/) applies to the data made available in this article, unless otherwise stated in a credit line to the data. 
Conclusion: Differential alteration of chemokines across the PPA variants is seen in both CSF and plasma. Importantly, these results suggest a role for neuroinflammation in these poorly understood sporadic disorders, and therefore also a potential future therapeutic target.

Keywords: Frontotemporal dementia, Primary progressive aphasia, Chemokines

\section{Background}

The primary progressive aphasias (PPA) are a rare group of disorders characterised by focal degeneration of the brain regions involved in language function [1] and fall within the frontotemporal dementia (FTD) spectrum. There are three main variants-the nonfluent or agrammatic variant (nfvPPA), the semantic variant (svPPA), and the logopenic variant (lvPPA) - distinguished by the language deficits with which they present as well as their neuroanatomical signatures [2]. Similarly, the predominant neuropathology underlying each of the variants usually differs, with nfvPPA most commonly a tauopathy such as progressive supranuclear palsy or corticobasal degeneration [3, 4], whilst svPPA is almost always a TDP-43 proteinopathy [5]. In contrast, lvPPA is not a frontotemporal lobar degeneration (FTLD) pathologically (i.e. not a primary tauopathy or TDP-43 proteinopathy) in most cases [6]; instead, the underlying pathology is usually that of Alzheimer's disease (AD), and lvPPA is often therefore considered an atypical language variant of $\mathrm{AD}$.

Recent studies have shown that neuroinflammation is an important pathophysiological factor in neurodegenerative disorders [7]. Although less research has been performed in FTD, molecular, pathological, and biomarker studies all suggest that inflammation is important here as well (reviewed in Bright et al. [8]). The process of neuroinflammation is complex and multistage but involves activation of glial cells, which in turn leads to upregulation of several proteins that help to guide the response. These include chemokines, a family of proteins that regulate leukocyte traffic but also have a number of other roles both within the immune system and outside, e.g. in development and synaptic transmission [9]. Only a few studies have so far investigated changes in chemokines in FTD spectrum disorders $[10,11]$, and so we aimed to assess this more thoroughly by using a panel of chemokines in the biofluids of a well-defined cohort of people from across the PPA spectrum, particularly focusing on changes centrally within the cerebrospinal fluid (CSF).

\section{Methods}

\section{Participants}

Thirty-six people with sporadic PPA and available CSF and plasma were consecutively recruited through the Longitudinal Investigation of FTD (LIFTD) study at
University College London (Table 1): 11 svPPA, 13 nfvPPA, and 12 lvPPA, diagnosed according to current consensus criteria [2]. All cases were negative for a C9orf72 expansion and mutations in any of the genes causative of FTD. 19 healthy controls were recruited over the same time period. All patients with lvPPA had a biomarker profile consistent with underlying Alzheimer's disease: mean (standard deviation) total tau/A 442 ratio (INNOTEST ${ }^{\circ}$, Fujirebio Europe N.V., Gent, Belgium) of 3.2 (2.2) with a range 1.2 to 8.3 where $>1$ is considered abnormal. All svPPA or nfvPPA participants and all controls had a ratio $<1$.

\section{Proximity Extension Assay panel}

Twenty chemokines were measured in the CSF and plasma of all participants using Proximity Extension Assay technology on the Olink $^{\circ}$ neuroinflammatory panel [12]. Briefly, samples were incubated with matched antibodies with DNA tags. When matched antibodies come in close proximity, DNA tags will only hybridise when the coupled antibodies match and then the sequence is amplified by qPCR. Results are expressed as normalised protein expression values. The chemokines measured were CCL2 (MCP-1), CCL3 (MIP-1a), CCL4 (MIP-1ß), CCL7 (MCP-3), CCL8 (MCP-2), CCL11 (eotaxin), CCL13 (MCP-4), CCL19, CCL20, CCL23, CCL25, CCL28, CX3CL1 (fractalkine), CXCL1, CXCL5, CXCL6, CXCL8 (IL-8), CXCL9, CXCL10, and CXCL11.

\section{Statistical analysis}

All statistical analyses were performed in STATA (v.16)) with the primary analysis focused on the results in the CSF. As this was an exploratory study, no correction for multiplicity was performed. The Shapiro-Wilk test was performed to determine the normality of distribution of each chemokine measure in each group.

Within the control group, Spearman correlation coefficients were assessed for each individual chemokine between their values and the age of participants at CSF collection. Sex differences were calculated using MannWhitney $U$ tests.

The levels of each chemokine in the CSF were then compared between groups using a linear regression model; bootstrapping with 1000 repetitions was used if the chemokine measures were not normal.

Spearman correlation coefficients were assessed for each individual chemokine between their values and 
Table 1 Demographics of participants in the study. PPA, primary progressive aphasia; SVPPA, semantic variant; nfv, nonfluent variant; IV, logopenic variant. N, number of participants. Values are shown as mean (standard deviation)

\begin{tabular}{lllll}
\hline & Controls & svPPA & nfvPPA & IvPPA \\
\hline$N$ & 19 & 11 & 13 & 12 \\
Age at CSF & $63.5(6.9)$ & $60.5(5.9)$ & $67.0(6.3)$ & $66.7(6.3)$ \\
Sex (\% male) & 47.4 & 54.5 & 53.8 & 50.0 \\
Disease duration at CSF & $\mathrm{N} / \mathrm{A}$ & $4.6(2.0)$ & $4.5(1.9)$ & $3.6(2.2)$ \\
A 4 42 & $999.9(235.4)$ & $879.7(259.5)$ & $845.6(318.3)$ & $439.8(159.4)$ \\
Total tau & $325.7(93.3)$ & $355.7(152.9)$ & $405.8(184.7)$ & $1206(555.4)$ \\
Total tau/Aß42 ratio & $0.3(0.1)$ & $0.4(0.1)$ & $0.5(0.3)$ & $3.2(2.2)$ \\
\hline
\end{tabular}

each of $\mathrm{A} \beta 42$ and total tau (t-tau) concentrations within the control and PPA groups.

In order to explore the relationship between chemokine values in CSF and plasma, Spearman correlation coefficients were assessed in each individual chemokine between CSF and plasma values within the control group as well as the disease groups.

The levels of each chemokine in the plasma were subsequently compared between groups using a linear regression model as previously; bootstrapping with 1000 repetitions was used if the chemokine measures were not normal.

\section{Results}

There were no significant differences between the groups in terms of either age at CSF collection or sex (Table 1).

Within the control group, no significant correlations were found between chemokines and age except for CXCL9 (rho $=0.55, p=0.016$ ) (Supplementary Table 1). No significant differences were found between chemokine values in males and females except in CCL28 where concentrations were lower in females $(0.4(0.1)$ versus $0.5(0.1)$ in males, $p=0.034)$.

Within CSF, three chemokines had $>80 \%$ of values across the groups below the lower limit of detection (CCL20 44/55, CCL13 53/55, CCL7 54/55) and were not assessed further. Seven of the remaining 17 chemokines showed a significant difference between groups. In both svPPA and nfvPPA, CCL19 and CXCL6 were decreased compared with controls (Fig. 1, Supplementary Table 2). CCL19 was additionally significantly decreased in both nfvPPA and svPPA compared with lvPPA, as was CXCL5, although this chemokine was decreased only in the nfvPPA group compared with controls (albeit with a borderline significant decrease in the svPPA group). In contrast, CCL2, CCL3 and CX3CL1 were increased in lvPPA compared with controls (and greater than nfvPPA for CCL2, and both nfvPPA and SvPPA for CX3CL1). CXCL1 was also increased in lvPPA compared with nfvPPA but not the other groups.
No correlations were found between A $\beta 42$ concentrations and values of any of the chemokines within the controls or the PPA groups except for a correlation in the nfvPPA group for CXCL6: rho $=0.58, p=0.033$ (Supplementary Figure 1). However, CX3CL1 was significantly correlated with $\mathrm{t}$-tau concentrations in the controls and each of the PPA groups (Supplementary Figure 1): controls rho $=0.51, p=0.025$, svPPA rho $=$ $0.64, p=0.035$, nfvPPA rho $=0.72, p=0.005$, lvPPA rho $=0.59, p=0.045$. None of the other chemokines correlated with t-tau across the groups but CCL4 was correlated in controls (rho $=0.59, p=0.033$ ), and CXCL5 was correlated in svPPA (rho $=0.65, p=0.029$ ).

In the chemokines that were found to be abnormal in CSF across groups, none of the CSF values were correlated with plasma values within the control group or any of the PPA groups. Only chemokines for which no significant group differences were seen showed a correlation between CSF and plasma values, both within the control group (CCL8: rho $=0.48, p=0.039$; CCL11: rho $=0.46 ; p=0.048 ;$ CCL25: rho $=0.53, p=0.021)$, the lvPPA group (CCL4: rho $=0.68, p=0.015$; CXCL8: rho $=0.79 ; p=0.002$ ), and the nfvPPA group (CCL4: rho $=$ $0.78, p=0.002$; CCL11: rho $=0.64 ; p=0.017$; CCL25: rho $=0.58, p=0.040 ;$ CXCL10: rho $=0.66, p=0.014)$. No correlations were seen in the svPPA group.

Within plasma, fewer significant differences were seen between groups (Supplementary Figure 1, Supplementary Table 3). However, CCL8 was higher in svPPA compared with controls and CCL13 was higher (with CCL20 lower) in nfvPPA compared with controls. In contrast, CCL3 and CCL19 were significantly lower in IvPPA compared with controls and the svPPA group (as well as the nfvPPA group for CCL3). Additionally, CCL4 was lower in lvPPA compared with nfvPPA but not with the other groups.

\section{Discussion}

We report chemokine levels in biofluids in PPA, showing differential alteration across the PPA variants. Interestingly, the direction of significant change 


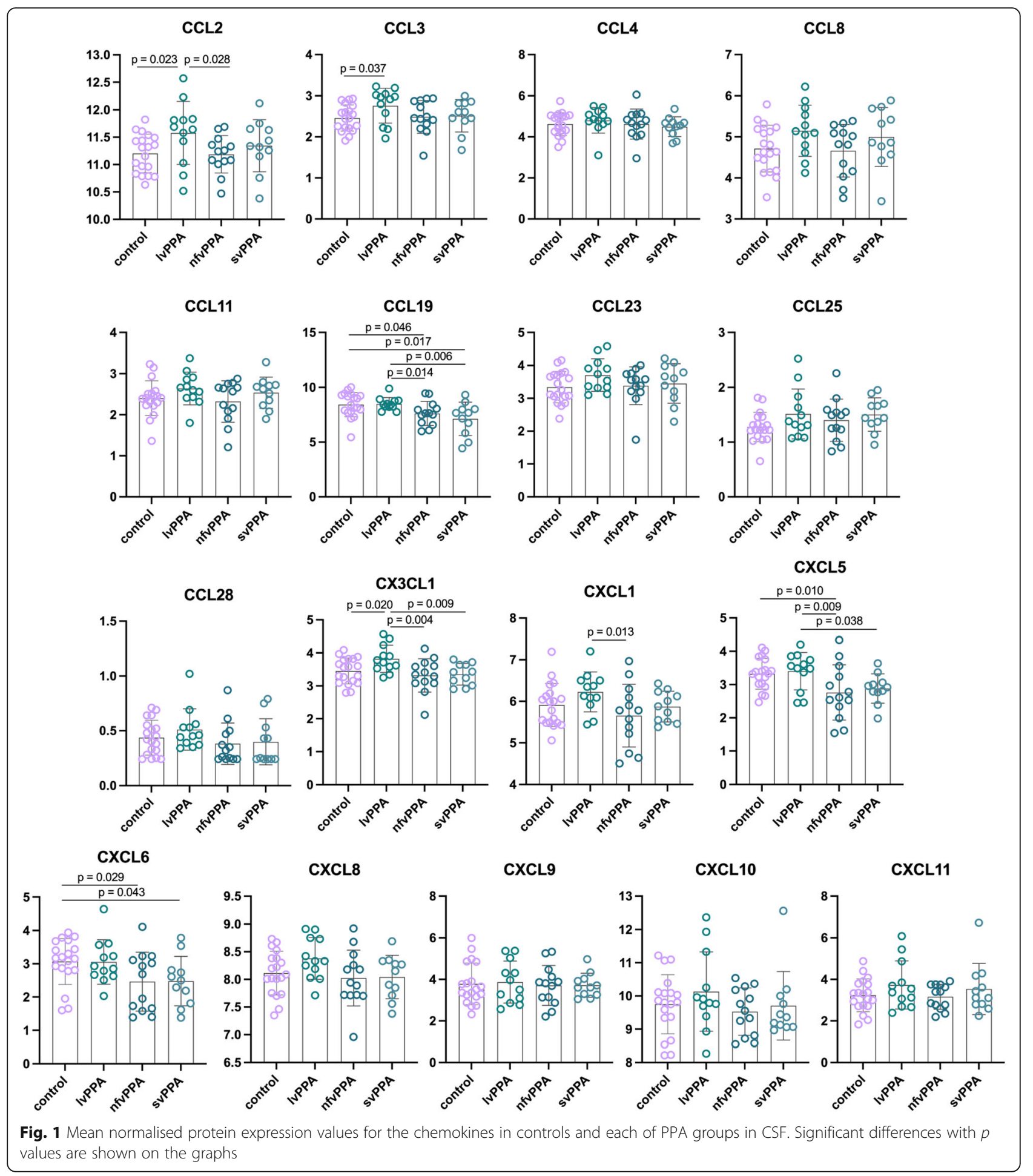

differed between those with underlying AD pathologically (lvPPA, where CSF values were increased and plasma values decreased) and those with FTLD (svPPA and nfvPPA, where CSF values were decreased, and for all but one chemokine, plasma values were increased). Comparing the CSF chemokine values with CSF biomarkers of amyloid and tau, CX3CL1, which was increased in IvPPA, was significantly correlated with $\mathrm{t}$-tau concentrations across all of the groups.

In keeping with lvPPA being associated with underlying $\mathrm{AD}$ pathology, the findings of increased CCL2, 
CCL3, and CX3CL1 in this group parallel previous studies in those with a typical AD clinical presentation [1318]. CCL2 (MCP-1) is expressed by neurons, astrocytes, and microglia and has previously been shown to be raised in the CSF of people with typical AD including those with mild cognitive impairment (MCI) $[13,14$, 19]. A further study also showed that CSF CCL2 concentrations correlated with the extent of brain atrophy and cognitive impairment in AD [15], with one report suggesting that higher levels were associated with more rapid progression from MCI to AD [16]. CCL3 (MIP-1a) is also expressed by neurons, astrocytes, and microglia [20], and this expression has been shown to be increased in the brains of people with $\mathrm{AD}$ as well as in mouse models [21, 22]. Here, we show increased levels of CCL3 in the CSF of the atypical AD phenotype lvPPA. Interestingly, we also show lower levels of CCL3 peripherally in the plasma, a finding also previously found by another group in serum [17]. The pathophysiological explanation for this difference between central and peripheral CCL3 levels remains unclear. Lastly, CX3CL1 (fractalkine) is produced by neurons, particularly in the hippocampus and cortex, and suppresses microglial activation [23]. Importantly, from an $\mathrm{AD}$ perspective, CX3CL1 has also been shown to be upregulated in the hippocampus during memory-associated synaptic plasticity [23]. Several studies have demonstrated significantly higher CSF concentrations of CX3CL1 in people with $\mathrm{MCI}$ and $\mathrm{AD}$ [21], with concentration being shown to differentiate MCI from controls with high sensitivity and specificity [18]. Our study also showed a strong correlation with ttau in lvPPA as well as in the controls and other groups, suggesting an association with increased neuronal damage particularly in the AD brain.

Although non-significant, a number of other chemokines showed a trend to an increase in lvPPA compared with controls, e.g. CCL23 $(p=0.068)$, increased concentrations of which have previously been shown to help predict progression from $\mathrm{MCI}$ to $\mathrm{AD}$ [24]. CXCL1 showed a significant increase in lvPPA compared with nfvPPA; this chemokine has also been shown to be increased in the CSF of people with $A D$ in a prior study [25].

Overall, this study highlights the important pathophysiological overlap of atypical variants of $\mathrm{AD}$ with the more typical variant. Here, we show that the central chemokine profile of change is similar in IvPPA to that found in amnestic AD.

In contrast, the two FTLD pathological forms of PPA show differential alterations of chemokines to lvPPA, with parallel decreases in CCL19, CXCL5, and CXCL6 in both svPPA and nfvPPA. This finding suggests that these are related to underlying FTLD pathology per se, rather than the individual proteinopathy, as svPPA
(TDP-43) and nfvPPA (tau) usually differ in their primary pathological cause. Unfortunately, little is known about alterations in chemokines levels in FTD so far. Only a few small studies have investigated chemokines, showing decreased levels of CCL5 (RANTES) and increased levels of CCL2, CXCL8, and CXCL10 in CSF [8, 10]. Previous studies have not investigated such an extensive set of chemokines in FTD across differential pathologies. CCL19, CXCL5, and CXCL6 are all expressed in the brain, although their roles are not clear. Nonetheless, CCL19 in particular has been studied in neuroinflammation-related diseases such as multiple sclerosis [26, 27], although here the levels were increased rather than decreased as found in our study. Whilst these are preliminary findings in nfvPPA and svPPA, they are important in signalling a role for neuroinflammation in these disorders. Little is currently known about why these sporadic diseases develop, and although a few studies have previously suggested a role for neuroinflammation in svPPA $[28,29]$, this study also suggests it is a relevant pathophysiological phenomenon in nfvPPA also. Interestingly, the levels of chemokines are generally decreased in svPPA and lvPPA compared to the increase seen in lvPPA-the reason for this is not clear: the inflammatory response to neurodegeneration is complex and studies investigating the response to inflammation including the involvement of the resolution pathway will be important.

Whilst central neuroinflammatory processes in neurodegenerative disorders are increasingly well-studied, fewer studies have investigated peripheral immune findings. There was generally poor correlation between CSF chemokine values and those in plasma within the controls, suggesting that peripheral values generally do not represent central levels. However, abnormal values were found in each of the PPA variants compared with controls (and for all but one measure, in the opposite direction to that found centrally in CSF). Certainly for svPPA, a previous study has showed an increase in systemic autoimmune disease compared with controls [29], but otherwise, little is known about peripheral inflammation in PPA, and more studies are required.

There are a number of limitations of the study. We did not have access to detailed behavioural or neuropsychometry data within the cohort, and it would be useful for future studies to investigate the correlation of clinical features with chemokine levels. The presence of comorbidities such as systemic disease, mood disorders, and cerebrovascular disease (including for the latter, the presence of white matter hyperintensities on MRI) were also not evaluated: their effect on chemokine levels would be important to clarify in further analyses. Whilst each group was of similar disease duration, participants were on average around 3 to 5 years into their illness. It 
would therefore be helpful to study both people very early in their clinical syndrome as well as to investigate longitudinal change in chemokines in PPA in order to understand the temporal relationship within the disease.

\section{Conclusions}

Overall, this study highlights the complex inflammatory response in the different variants of PPA and shows clear differences between those with AD and FTLD pathology. Such biomarkers may be helpful in future trials for a number of reasons including assessing the extent of neuroinflammation present [30], although not for individually classifying the different forms of PPA. Our results establish a baseline for further study of the role of chemokines in PPA with the potential role of neuroinflammation as a therapeutic target in these sporadic disorders an important area of future research.

\begin{abstract}
Abbreviations
PPA: Primary progressive aphasia; FTD: Frontotemporal dementia; nfvPPA: Nonfluent/agrammatic variant PPA; svPPA: Semantic variant PPA; IVPPA: Logopenic variant PPA; FTLD: Frontotemporal lobar degeneration; TDP-43: TAR DNA-binding protein 43; AD: Alzheimer's disease; CSF: Cerebrospinal fluid; C9orf72: Chromosome 9 open reading frame 72; QPCR: Quantitative Polymerase Chain Reaction; CCL2 (MCP-1): C-C motif chemokine 2 (Monocyte chemoattractant protein 1); CCL3 (MIP-1a): C-C motif chemokine 3 (Macrophage inflammatory protein 1 alpha); CCL4 (MIP1B): C-C motif chemokine 4 (Macrophage inflammatory protein 1 beta); CCL7 (MCP-3): C-C motif chemokine 7 (Monocyte chemoattractant protein 3); CCL8 (MCP-2): C-C motif chemokine 8 (Monocyte chemoattractant protein 2): CCL11: Eotaxin; CCL13 (MCP-4): C-C motif chemokine 13 (Monocyte chemoattractant protein 4); CCL19: C-C motif chemokine 19; CCL20: C-C motif chemokine 20; CCL23: C-C motif chemokine 23; CCL25: C-C motif chemokine 25; CCL28: C-C motif chemokine 28; CX3CL1: Fractalkine; CXCL1: Growth-regulated alpha protein; CXCL5: C-X-C motif chemokine 5; CXCL6: C-X-C motif chemokine 6; CXCL8 (IL-8): Interleukin 8; CXCL9: C-X-C motif chemokine 9; CXCL10: C-X-C motif chemokine 10; CXCL11: C-X-C motif chemokine 11; MCl: Mild cognitive impairment
\end{abstract}

\section{Supplementary Information}

The online version contains supplementary material available at https://doi. org/10.1186/s12974-021-02247-3.

Additional file 1: Supplementary Figure 1. Mean normalized protein expression values for the chemokines in controls and each of PPA groups in plasma. Significant differences with $p$ values are shown on the graphs.

Additional file 2: Supplementary Table 1. Spearman correlation coefficients and $p$-values comparing chemokines with age at CSF collection within the control group. Supplementary Table 2. Mean (standard deviation) normalized protein expression values for the chemokines in controls and each of PPA groups in CSF. Mean differences between the PPA groups and controls along with 95\% confidence intervals and $p$ values (significant in bold) are shown underneath. N/A = not assessed $>80 \%$ values were below the lower limit of detection.

Supplementary Table 3. Mean (standard deviation) normalized protein expression values for the chemokines in controls and each of PPA groups in plasma. Mean differences between the PPA groups and controls along with 95\% confidence intervals and $p$ values (significant in bold) are shown underneath. $\mathrm{N} / \mathrm{A}=$ not assessed $>80 \%$ values were below the lower limit of detection.

\section{Authors' contributions}

ASE and JDR analysed and interpreted the data and wrote the initial draft of the manuscript. IOCW and ISJ collected and processed the samples from participants. All authors read and approved the final manuscript.

\section{Funding}

The Dementia Research Centre is supported by Alzheimer's Research UK, Alzheimer's Society, Brain Research UK, and The Wolfson Foundation. This work was supported by the NIHR UCL/H Biomedical Research Centre, the Leonard Wolfson Experimental Neurology Centre (LWENC) Clinical Research Facility, and the UK Dementia Research Institute, which receives its funding from UK DRI Ltd, funded by the UK Medical Research Council, Alzheimer's Society and Alzheimer's Research UK. ASE is supported by the UK Dementia Research Institute which receives its funding from DRI Ltd, funded by the UK Medical Research Council, Alzheimer's Society and Alzheimer's Research UK. IJS is supported by the Alzheimer's Association. IOCW was supported by an MRC Clinical Research Training Fellowship (MR/M018288/1). JDW receives funding support from the Alzheimer's Society, Alzheimer's Research UK, and NIHR UCLH Biomedical Research Centre. HZ is a Wallenberg Scholar supported by grants from the Swedish Research Council (\#2018-02532), the European Research Council (\#681712), Swedish State Support for Clinical Research (\#ALFGBG-720931), the Alzheimer Drug Discovery Foundation (ADDF), USA (\#201809-2016862), the AD Strategic Fund and the Alzheimer's Association (\#ADSF-21-831376-C, \#ADSF-21-831381-C and \#ADSF-21-831377C), the Olav Thon Foundation, the Erling-Persson Family Foundation, Stiftelsen för Gamla Tjänarinnor, Hjärnfonden, Sweden (\#FO2019-0228), the European Union's Horizon 2020 research and innovation programme under the Marie Skłodowska-Curie grant agreement No 860197 (MIRIADE), and the UK Dementia Research Institute at UCL. JDR has received funding from an MRC Clinician Scientist Fellowship (MR/M008525/1) and an NIHR Rare Disease Translational Research Collaboration (BRC149/NS/MH); his work is also supported by the MRC UK GENFI grant (MR/M023664/1), the Bluefield Project, and the JPND GENFI-PROX grant (2019-02248)

\section{Availability of data and materials}

The datasets used and/or analysed during the current study are available from the corresponding author on reasonable request.

\section{Declarations}

Ethics approval and consent to participate

The London Queen Square Ethics committee approved the study. The study complies with the Declaration of Helsinki.

\section{Consent for publication}

All participants provided written informed consent at enrolment including consent to publication.

\section{Competing interests}

$\mathrm{HZ}$ has served at scientific advisory boards for Eisai, Denali, Roche Diagnostics, Wave, Samumed, Siemens Healthineers, Pinteon Therapeutics, Nervgen, AZTherapies, and $\operatorname{CogRx}$, has given lectures in symposia sponsored by Cellectricon, Fujirebio, Alzecure, and Biogen, and is a co-founder of Brain Biomarker Solutions in Gothenburg AB (BBS), which is a part of the GU Ventures Incubator Program. JDR has served on medical advisory boards and undertaken consultancy for Alector, Arkuda Therapeutics, Wave Life Sciences, and Prevail Therapeutics. He has also undertaken consultancy for UCB, AC Immune, Astex Pharmaceuticals, Biogen, Takeda and Eisai.

\section{Author details}

${ }^{1}$ UK Dementia Research Institute at University College London, UCL Queen Square Institute of Neurology, University College London, London, UK. ${ }^{2}$ Dementia Research Centre, Department of Neurodegenerative Disease, UCL Queen Square Institute of Neurology, University College London, London WC1N 3BG, UK. ${ }^{3}$ Department of Psychiatry and Neurochemistry, The Sahlgrenska Academy at the University of Gothenburg, Mölndal, Sweden. ${ }^{4}$ Clinical Neurochemistry Laboratory, Sahlgrenska University Hospital, Mölndal, Sweden. 
Received: 3 April 2021 Accepted: 24 August 2021

Published online: 03 October 2021

\section{References}

1. Marshall CR, Hardy CJD, Volkmer A, Russell LL, Bond RL, Fletcher PD, et al. Primary progressive aphasia: a clinical approach. J Neurol. 2018;265(6):147490. https://doi.org/10.1007/s00415-018-8762-6.

2. Gorno-Tempini ML, Hillis AE, Weintraub S, Kertesz A, Mendez M, Cappa SF, et al. Classification of primary progressive aphasia and its variants. Neurology. 2011;76(11):1006-14. https://doi.org/10.1212/WNL.0b013e318211 $03 e 6$

3. Graff-Radford J, Duffy JR, Strand EA, Josephs KA. Parkinsonian motor features distinguish the agrammatic from logopenic variant of primary progressive aphasia. Park Relat Disord. 2012;18(7):890-2. 6.

4. Kremen SA, Mendez MF, Tsai PH, Teng E. Extrapyramidal signs in the primary progressive aphasias. Am J Alzheimers Dis Other Demen. 2011;26(1): 72-7. https://doi.org/10.1177/1533317510391239.

5. Hodges JR, Patterson K. Semantic dementia: a unique clinicopathological syndrome. Lancet Neurol. 2007;6(11):1004-14. https://doi.org/10.1016/S14 74-4422(07)70266-1.

6. Henry ML, Gorno-Tempini ML. The logopenic variant of primary progressive aphasia. Curr Opin Neurol. 2010;23(6):633-7. https://doi.org/10.1097/WCO. ob013e32833fb93e.

7. Chen WW, Zhang X, Huang WJ. Role of neuroinflammation in neurodegenerative diseases (Review). Mol Med Rep. 2016;13(4):3391-6. https://doi.org/10.3892/mmr.2016.4948.

8. Bright F, Werry EL, Dobson-Stone C, Piguet O, Ittner LM, Halliday GM, et al. Neuroinflammation in frontotemporal dementia. Nat Rev Neurol. 2019;15(9): 540-55. https://doi.org/10.1038/s41582-019-0231-z.

9. Miller RJ, Rostene W, Apartis E, Banisadr G, Biber K, Milligan ED, et al. Chemokine action in the nervous system. J Neurosci. 2008;28(46):11792-5. https://www.doi.org/10.1523/jneurosci.3588-08.2008

10. Galimberti D, Bonsi R, Fenoglio C, Serpente M, Cioffi SMG, Fumagalli G, et al. Inflammatory molecules in frontotemporal dementia: cerebrospinal fluid signature of progranulin mutation carriers. Brain Behav Immun. 2015:49: 182-7. https://doi.org/10.1016/j.bbi.2015.05.006.

11. Galimberti D, Venturelli E, Fenoglio C, Guidi I, Villa C, Bergamaschini L, et al. Intrathecal levels of IL-6, IL-11 and LIF in Alzheimer's disease and frontotemporal lobar degeneration. J Neurol. 2008;255(4):539-44. https://doi. org/10.1007/s00415-008-0737-6.

12. Assarsson $E$, Lundberg M, Holmquist G, Björkesten J, Thorsen SB, Ekman D, et al. Homogenous 96-plex PEA immunoassay exhibiting high sensitivity, specificity, and excellent scalability. PLoS One. 2014;9(4). https://doi.org/1 0.1371/journal.pone.0095192.

13. Shen XN, Niu LD, Wang YJ, Cao XP, Liu Q, Tan L, et al. Inflammatory markers in Alzheimer's disease and mild cognitive impairment: a meta-analysis and systematic review of 170 studies. J Neurol Neurosurg Psychiatry. 2019;90(5): 590-8. https://doi.org/10.1136/jnnp-2018-319148.

14. Galimberti D, Schoonenboom N, Scheltens P, Fenoglio C, Bouwman F, Venturelli $\mathrm{E}_{\text {, et }}$ al. Intrathecal chemokine synthesis in mild cognitive impairment and Alzheimer disease. Arch Neurol. 2006;63(4):538-43. https:// doi.org/10.1001/archneur.63.4.538.

15. Kimura A, Yoshikura N, Hayashi Y, Inuzuka T. Cerebrospinal fluid C-C motif chemokine ligand 2 correlates with brain atrophy and cognitive impairment in Alzheimer's disease. J Alzheimers Dis. 2017;61(2):581-8. https://doi.org/1 $0.3233 / J A D-170519$

16. Westin K, Buchhave $P$, Nielsen $H$, Minthon L, Janciauskiene $S$, Hansson O. CCL2 is associated with a faster rate of cognitive decline during early stages of Alzheimer's disease. PLoS One. 2012;30(1):7(1). https://doi.org/10.1371/ journal.pone.0030525.

17. Geppert AM, Losy J, Przedpelska-Ober E, Kozubski W. CCL3 correlates with the number of mood disturbances and personality changes in patients with Alzheimer's disease. Psychiatry Res. 2010;176(2-3):261-4. https://doi.org/10.1 016/j.psychres.2009.02.007.

18. Kulczyńska-Przybik A, Słowik A, Mroczko P, Borawski B, Groblewska M, Borawska R, et al. Cerebrospinal fluid and blood CX3CL1 as a potential biomarker in early diagnosis and prognosis of dementia. Curr Alzheimer Res. 2020;9(8):17-721. https://doi.org/10.2174/1567205017666201109095657.

19. Nordengen $K$, Kirsebom BE, Henjum $K$, Selnes $P$, Gísladóttir $B$, Wettergreen $M$, et al. Glial activation and inflammation along the Alzheimer's disease continuum. J Neuroinflammation. 2019;16(1). https://doi.org/10.1186/s12 974-019-1399-2.

20. Xia M, Qin S, Wu L, Mackay CR, Hyman BT. Immunohistochemical study of the $\beta$-chemokine receptors CCR3 and CCR5 and their ligands in normal and Alzheimer's disease brains. Am J Pathol. 1998;153(1):31-7. https://doi.org/1 0.1016/S0002-9440(10)65542-3.

21. Azizi G, Khannazer N, Mirshafiey A. The potential role of chemokines in Alzheimer's disease pathogenesis. Am J Alzheimers Dis Demen. SAGE Publications Inc. 2014;29:415-25. https://doi.org/10.1177/1533317513518651

22. Martin E, Boucher C, Fontaine B, Delarasse C. Distinct inflammatory phenotypes of microglia and monocyte-derived macrophages in Alzheimer's disease models: effects of aging and amyloid pathology. Aging Cell. 2017;16(1):27-38. https://doi.org/10.1111/acel.12522.

23. Sheridan GK, Wdowicz A, Pickering M, Watters $O$, Halley $P$, O'Sullivan NC, et al. CX3CL1 is up-regulated in the rat hippocampus during memoryassociated synaptic plasticity. Front Cell Neurosci. 2014;8:233. https://doi. org/10.3389/fncel.2014.00233

24. Faura J, Bustamante A, Penalba A, Giralt D, Simats A, Martínez-Sáez E, et al. CCL23: A chemokine associated with progression from mild cognitive impairment to Alzheimer's disease. J Alzheimer's Dis. 2020;73(4):1585-95. https://doi.org/10.3233/JAD-190753.

25. Craig-Schapiro R, Kuhn M, Xiong C, Pickering EH, Liu J, Misko TP, et al. Multiplexed immunoassay panel identifies novel CSF biomarkers for Alzheimer's disease diagnosis and prognosis. Bush Al, editor. PLoS One. 2011;6(4):e18850. https://doi.org/10.1371/journal.pone.0018850

26. Krumbholz M, Theil D, Steinmeyer F, Cepok S, Hemmer B, Hofbauer M, et al. CCL19 is constitutively expressed in the CNS, up-regulated in neuroinflammation, active and also inactive multiple sclerosis lesions. J Neuroimmunol. 2007;190(1-2):72-9. https://doi.org/10.1016/j.jneuroim.2007. 07.024

27. Lepennetier G, Hracsko Z, Unger M, Van Griensven M, Grummel V, Krumbholz M, et al. Cytokine and immune cell profiling in the cerebrospinal fluid of patients with neuro-inflammatory diseases. J Neuroinflammation. 2019;16(1, 1). https://doi.org/10.1186/s12974-019-1601-6.

28. Heller C, Chan E, Foiani MS, Todd E, Russell LL, Greaves CV, et al. Plasma glial fibrillary acidic protein and neurofilament light chain are measures of disease severity in semantic variant primary progressive aphasia. J Neurol Neurosurg Psychiatry. 2020:jnnp-2020-325085. https://doi.org/10.1136/jnnn-2 020-325085

29. Miller ZA, Rankin KP, Graff-Radford NR, Takada LT, Sturm VE, Cleveland CM, et al. TDP-43 frontotemporal lobar degeneration and autoimmune disease. $J$ Neurol Neurosurg Psychiatry. 2013;84(9):956-62. https://doi.org/10.1136/ jnnp-2012-304644.

30. Swift IJ, Sogorb-Esteve A, Heller C, Synofzik M, Otto M, Graff C, et al. Fluid biomarkers in frontotemporal dementia: past, present and future. J Neurol Neurosurg Psychiatry. BMJ Publishing Group; 2020. 2021;92(2):204-15. https://doi.org/10.1136/jnnp-2020-323520.

\section{Publisher's Note}

Springer Nature remains neutral with regard to jurisdictional claims in published maps and institutional affiliations.

Ready to submit your research? Choose BMC and benefit from:

- fast, convenient online submission

- thorough peer review by experienced researchers in your field

- rapid publication on acceptance

- support for research data, including large and complex data types

- gold Open Access which fosters wider collaboration and increased citations

- maximum visibility for your research: over $100 \mathrm{M}$ website views per year

At BMC, research is always in progress.

Learn more biomedcentral.com/submissions 